Kürzere Beiträge

\title{
Die Spitalversorgung im Emmental
}

\author{
Von Marta Meyer-Salzmann
}

Im Emmental taucht der Name Spital erstmals im Jahr 1225 auf $^{1}$. Der Deutsche Orden baute nach einer Stiftung des Edlen Lütold von Sumiswald dort ein Schloß als Ordenshaus und Spital. Das Gebäude ist heute noch erhalten und darin der Krankensaal mit Seitenkapelle. Diese Spitalanlage reiht sich unter die seltenen noch vorhandenen frühen europäischen Spitäler diesseits der Alpen ein ${ }^{2}$. Nach wechselnden Schicksalen beherbergt heute das Schloß ein Pflegeheim.

Im Städtchen Burgdorf ermöglichten 50 Jahre nach der Sumiswalder Schenkung die Grafen von Kyburg ein Spital, an dessen Krankensaal ebenfalls eine Kapelle angegliedert war. Es stand fast 600 Jahre den bettlägerigen Kranken zur Verfügung. Einzelne gotische Merkmale der Kapelle sind noch erhalten, aber nicht mehr gut zugänglich, denn das Gebäude dient heute als Schlachthaus. Ein weiteres Spital in Burgdorf-die Schenkung der Witwe eines begüterten Schmiedes - nahm vor allem Reisende und Pfründer auf ${ }^{3}$.

Es gab im Emmental sieben Siechenhäuser. Dort wurden erst Aussätzige, dann Pflegebedürftige anderer Art von Wundärzten besorgt. Das Siechenhaus in Burgdorf war für Invalide noch bis Ende des 18. Jahrhunderts in Betrieb, dasjenige von Huttwil bis ins 19. Jahrhundert; alle andern gingen weit früher ein ${ }^{4}$. Das Gebäude in Burgdorf, ein schönes Sandsteinhaus mit gotischen Stilmerkmalen, und die dazugehörende freistehende gotische Kapelle sind Schmuckstücke der Gegend. Im ehemaligen Siechenhaus ist heute ein Privatkindergarten untergebracht; die Kapelle wird oft für Hochzeiten beansprucht.

In Trub und Rüegsau sorgten zwei kleine Benediktinerklöster für Hilfsbedürftige, in Burgdorf das Antonierhaus und das Barfüßerkloster, ohne daß sie den Charakter eines Spitals annahmen ${ }^{5}$. Das Kloster Trub war um 1125 gegründet worden. Die Klöster gingen zur Reformationszeit ein. Auch der Deutsche Orden zog von Sumiswald fort; das Schloß nahm den Landvogteisitz auf ${ }^{6}$. In der zweiten Hälfte des Mittelalters besaß also die Landschaft verhältnismäßig zahlreiche Häuser, die in irgendeiner Weise 
Hilfe für Kranke boten, die nicht im Familienkreis betreut werden konnten.

Die Krankenversorgung mußte nach der Reformation von den Behörden neu geordnet werden. Ihre Fürsorge galt den Unbemittelten. Kirchgemeinden und Gütergemeinden veranlaßten den sogenannten «Umgang»; das heißt, Invalide und Kranke wurden den Güterbesitzern abwechslungsweise zur Verköstigung oder auch zur Betreuung zugewiesen ?

Letzte Nothilfe für die Gemeinden war aber das von Anna Seiler 1354 gestiftete Spital in Bern, das spätere Inselspital und nunmehrige Universitätsspital. Im bernischen Ratsmanual steht 1535: «Dem uss dem Emental im Seilerinspital umb gotzwillen das lam bein abnemen.» ${ }^{8}$ Auch die dem Inselspital angegliederten Zellen für Geisteskranke bildeten eine Zufluchtsmöglichkeit, falls dort Platz vorhanden war ${ }^{9}$.

Im Emmental gab es allerdings im 17. und 18. Jahrhundert je eine Privatklinik für Geisteskranke. Beide wurden von Wundärzten geführt, die sich um die Heilung dieser Kranken bemühten ${ }^{10}$. Auch einzelnen Privatleuten gaben die Behörden Geisteskranke zur Aufsicht ${ }^{11}$.

Das Kloster Thorberg bei Krauchthal, geographisch dem Mittelland, politisch dem Amt Burgdorf zugehörend, nahm nach der Reformation Pfründer auf. Unter ihnen befanden sich Pfarrer und Lehrer. In einem Gebäude wurde ein Spital für Alte und Gebrechliche errichtet; in die ehemaligen Mönchszellen kamen «schwierige Leute», das heißt Geisteskranke und Epileptiker, vorwiegend aus den Ämtern Konolfingen und Burgdorf ${ }^{12}$.

Badehäuser und Mineralquellen des Emmentals dienten Heilung Suchenden ${ }^{13}$. Für Badekuren außerhalb des Emmentals zahlten die Behörden, wo es ihnen notwendig erschien, einen Beitrag ${ }^{14}$. Im 18. Jahrhundert taten sich emmentalische Wundärzte in der Chirurgie hervor. Es gab vereinzelte, die auch in der Stadt Bern praktizierten ${ }^{15}$. Operationen wurden meistens in Privathäusern durchgeführt ${ }^{16}$. Zwei Belege von Kaiserschnitten waren aus Langnau und Sumiswald zu ermitteln ${ }^{17}$. Ab und zu nahmen Wundärzte Patienten zur Pflege in ihr Haus auf und führten eine Art Privatklinik ${ }^{18}$. Die Behörden zahlten oft den Wundärzten für die Versorgung Armer namhafte Beiträge ${ }^{19}$.

Für unbemittelte Schwerkranke wurde auch immer wieder um Aufnahme ins Inselspital nachgesucht ${ }^{20}$. Sie erhielten dort kostenlose Behandlung und Pflege. Das Inselspital war häufig überfüllt ${ }^{21}$; es brachte für die Gemeindebehörden die ersehnte finanzielle Entlastung. Bemittelte konnten sich vom Wundarzt helfen lassen. 
Im ersten Drittel des 19. Jahrhunderts nahmen die Bevölkerung und die Arbeitslosigkeit zu. Damals entstanden Gemeindespitäler, die der Volksmund Spittel nannte. Es waren Armen-, Arbeits- und Erziehungsanstalten, worin auch Kranke, Invalide und alleinstehende Wöchnerinnen vom Anstaltsarzt betreut wurden ${ }^{22}$. Die Gemeinden standen vor schwierigen Aufgaben und schweren Lasten. Eine solche Anstalt befand sich auch in Sumiswald im ehemaligen Ordenshaus und Spital des Deutschen Ordens. Nach 300 Jahren wurden dort wieder in beschränktem Umfang Pflegebedürftige untergebracht. Durch ein Dekret der bernischen Regierung von $1834 \mathrm{kam}$ es in dieser Anstalt und auch in Langnau zur Errichtung sogenannter Notfallstuben mit je vier Betten, die der bernische Staat mit Geld unterstützte ${ }^{23}$. Nun konnten endlich unbemittelte Akuterkrankte sofort der Behandlung und Pflege zugeführt werden.

Die Notfallstuben bildeten die Vorläufer der Bezirksspitäler. Diese gelangten durch vereinsähnliche Zusammenschlüsse von Gemeinden zur Ausführung: 1877 in Burgdorf ${ }^{24}, 1879$ in Sumiswald ${ }^{25}, 1881$ in Langnau ${ }^{26}$, und im emmentalischen Gebiet folgten vier weitere. Als Sumiswald sein Gesuch um die Eröffnung eines Spitals an die bernische Sanitätsdirektion einreichte, wurde ein Haus allein für Kranke, ein Badezimmer und ein Operationszimmer für die Landbevölkerung als angemessen erkannt. Die Spitäler sollten Unbemittelten dienen.

In Sumiswald wurde in einem schönen neuen Haus mit 14 Betten begonnen; Platz war für 20 vorgesehen. Bei der Eröffnung konnte der Arzt über ein gut dotiertes zeitgemäßes Instrumentarium verfügen; ein Operationstisch für 150 Franken und eine Operationsbettstelle für 48 Franken gehörten dazu; auch ein Elektrisierapparat kam bald in Gebrauch. Verunfallte wurden dort eingeliefert und häufig Wunden gepflegt. Der Luftröhrenschnitt wurde öfters ausgeführt. Die inneren Krankheiten betrafen nach Berichten aus Burgdorf: Lungen- und Brustfellentzündung, Lungentuberkulose, Entzündung des Herzmuskels, Bauchfellentzündung, Verdauungsschwäche, Darmkatarrh, Typhus, Venenentzündung und verschiedene Arten von Rheumatismus.

Nach mündlicher Überlieferung wurde 1902 eine Kropfoperation in der Praxis des Arztes in Sumiswald durchgeführt. Im Jahresbericht von 1923 des Bezirksspitals in Huttwil steht, daß im Privathaus nur noch selten operiert werde, der Patient komme ins Spital. Die Bezirksspitäler waren erweitert worden und standen nun längst auch Selbstzahlenden offen.

In Burgdorf war 1913, in Sumiswald 1920 ein Röntgenapparat in 
Betrieb. Als derjenige in Sumiswald fünf Jahre später technisch schon veraltet war, schaffte der Spitalarzt Dr. Werner Frölich auf eigene Kosten ein neues Gerät an und stellte es dem Spital unentgeltlich zur Verfügung.

Im Jahresbericht von Burgdorf steht 1904, daß bei Bruchoperationen die Methoden nach Kocher angewendet wurden, bei einer Gastroenterostomie jene nach Kocher und Roux. 1908 wird vermerkt, daß stets mit trockenen, sterilen Gummihandschuhen operiert werde. 1908 wurde in Sumiswald erstmals ein Blinddarm operiert, 1910 eine Gallenblase. Verschiedene Unterleibsoperationen folgten, 1918 ein Eingriff bei einer Bauchhöhlenschwangerschaft sowie ein erster Kaiserschnitt. 1930 führte Dr. Frölich in Sumiswald trotz seiner umfangreichen Privatpraxis, die Hausbesuche in weitem Umkreis erforderte, 486 Operationen von ungewöhnlicher Vielseitigkeit aus.

In Langnau war als Chirurg Prof. Dr. Anton Fonio von 1914 bis 1944 tätig. Er führte dort die Bluttransfusion ein. Eine spektakuläre Operation war 1922 die Einsetzung einer künstlichen Speiseröhre in Form eines Hautschlauches. Die Patientin konnte nach Verheilung der Wunde wieder wie ein normaler Mensch essen und trinken. Fonio war es auch, der in Langnau ein Verfahren zur Herstellung von Blutkonserven ausarbeitete.

Die Bezirksspitäler entwickelten nach und nach gesonderte Abteilungen, erst für innere Medizin und Chirurgie. Die meisten Sonderabteilungen weist heute das Spital in Burgdorf auf. Sein großes Einzugsgebiet umfaßt auch Gemeinden des Oberaargaus. Seit 1908 besteht dort eine Augenabteilung, die einzige im Emmental. Der Ausbau der Medizin verlangt gegenseitige Absprachen. So steht die in Burgdorf angestellte Anästhesistin auch den Spitälern in Langnau und Sumiswald zur Verfügung. Prof. Dr. Hans Stirnemann, der heutige chirurgische Chefarzt in Burgdorf, ist ein großer Förderer der guten Zusammenarbeit mit andern Bezirksspitälern. Das Spital in Burgdorf ist 1979 als Regionalspital eingestuft worden. Und das Inselspital oder Universitätsspital mit seinen Einrichtungen und Spezialärzten steht den Emmentalern weiterhin treu zur Seite.

Es handelt sich bei dieser Darstellung um Ergebnisse aus meiner Geschichte der Medizin im Emmental, Festschrift zum hundertjährigen Jubiläum des Bezirksspitals in Sumiswald 1979 (hrsg. vom Bezirksspital in Sumiswald). 


\section{Anmerkungen}

${ }^{1}$ Mülinen, Egbert Friedrich von, Die weltichen und geistlichen Herren des Emmentals, Bern 1872, S. 18-19, 58-83. Die Urkunden über die Schenkung des Edlen Lütold von Sumiswald befinden sich im Staatsarchiv Stuttgart, Kopien im Staatsarchiv Bern.

Der Deutsche Orden als Macht und im Dienst an Kranken:

Acht Jahrhunderte Deutscher Orden, hrsg. von P.Dr. Klemens Wieser O.T., Bad Godesberg 1967, S.38, 103, 166-167, 170;

Probst, Christian, Der Deutsche Orden und sein Medizinalwesen in Preußen, Bad Godesberg 1969, S. 24, 99;

Brunner, Conrad, Über Medizin und Krankenpflege im Mittelalter in schweizerischen Landen (Veröffentlichungen der Schweiz. Ges. für Geschichte der Medizin und der Naturwissenschaften, Nr. 1), Zürich 1922, S. 126-131;

Imobersteg, Jakob, Das Emmental nach Geschichte, Land und Leuten, Bern 1886, S.29, $32-35,40$.

Im Museum Schatzkammer des Deutschen Ordens in Wien ist auf einer großen Wandkarte als südlichste Gründung eines Ordenshauses Sumiswald eingezeichnet.

2 Vgl. Craemer, Ulrich, Das Hospital als Bautyp des Mittelalters, Köln 1963.

${ }^{3}$ Geiser, Karl, Von den Alemannen bis zum Übergang Burgdorfs an Bern 1384. In: Heimatbuch des Amtes Burgdorf und der Kirchgemeinden Utzenstorf und Bätterkinden, 1938, S.94, 115;

Vatter, Max, Das religiöse und kirchliche Leben, einschließlich des Fürsorgewesens. Ibid., S. 346;

Grimm, August, Naturalverpflegung «Dürftiger Wanderer». In: Burgdorfer Jahrbuch 1953.

4 Über Leprakranke und ihre Häuser:

Koelbing, Huldrych M., Trümpy, Hans, et al., Beiträge zur Geschichte der Lepra (Zürcher medizingesch. Abhandl., Neue Reihe Nr.93), Zürich 1972;

Nüscheler, Arnold, Die Siechenhäuser in der Schweiz. In: Archiv für Schweiz. Geschichte 15 (Zürich 1866), S. 208;

Lachat, Paul, Seelsorge und Kapelle bei den Siechen in Burgdorf. In: Burgdorfer Jahrbuch 1957;

Nyffeler, Ernst, Heimatkunde von Huttwil, neub. und erg., Huttwil 1915, S.213;

Vogel, Niklaus, Oberdießbach. Die Geschichte eines Dorfes. Oberdießbach 1960, S.83;

Thüer, Hans Rudolf, Der Aussatz. In: Heimatkunde des Wiggertales 1977, H. 35, hrsg. von der Heimatvereinigung Wiggertal, Willisau 1977, S.118-125.

${ }^{5}$ Laedrach, Walter, Das Kloster Trub und die Hoheit über das Trubertal, Diss. Bern 1921;

Würgler, Hans, Heimatkunde von Rüegsau, Rüegsau 1965, S. 15-25;

Kasser, Hermann, Das Bernbiet ehemals und heute, Bd.1, Bern 1905, S. 37, 113;

Imobersteg (siehe Anm. 1), S. 6, 20-24;

Vatter (siehe Anm.3), S. 329-337.

${ }^{6}$ Mülinen (siehe Anm. 1), S. 78-80.

7 Zuber, Jakob, Die Armenanstalt der Einwohnergemeinde Sumiswald, 1812-1912, Sumiswald 1913, S. 6.

Vatter (siehe Anm. 3), S.409-410.

Gesnerus 3/4 (1980) 
${ }^{8}$ Rennefahrt, Hermann, und Hintzsche, Erich, Sechshundert Jahre Inselspital, 1354-1954, Bern 1954, S. 204.

${ }^{9}$ Ibid., S. 67, 68, 206, 207.

${ }^{10}$ Rubi, Christian, Die Irrenärzte Neukommet aus Signau. In: Archiv des Hist. Vereins des Kantons Bern XXXIX, H. 2 (Festschrift für Richard Feller), Bern 1948;

Nyffeler (siehe Anm. 4), S.315, 316.

${ }_{11}$ Rubi (siehe Anm. 10), S. 325.

12 Schweingruber, Max, Heimatbuch Krauchthal/Thorberg, Krauchthal 1971, S. 353, 370, 397, $434,435,451$.

${ }^{13}$ Meyer-Salzmann (siehe oben), S.32, 33, 37, 38, 42, 59, 60 .

${ }^{14}$ Amtsrechnungen der Landvogtei Trachselwald (Grundbuchamt Trachselwald), 1629, 1689.

${ }^{15}$ Werder, Ernst, Aus der Geschichte des Arzneiwesens im Amt Konolfingen. In: Berner Zeitschrift für Geschichte und Heimatkunde, H.2, Bern 1947, S. 88;

Meyer-Salzmann, Marta, Michel Schüppach, 1707-1781. Ein Höhepunkt handwerklicher Heilkunst (Berner Heimatbuch 126), Bern 1980, 1. Kapitel. Neun Lehrbriefe im Wortlaut erwähnen die Chirurgie mit einzelnen ausgeführten Operationen.

${ }^{16}$ Ibid., Lehrbrief 11.

${ }^{17}$ Grunau, Gustav, Hrsg. der Blätter für Bernische Geschichte, Kunst und Altertumskunde, Bd.IX, Bern 1913, S.269: «In der Gemeinde Langnau befand sich ein Mägdlein von Acht Jahren und zehn Monaten Alters schwanger und kam zur Gnißt [Geburt]. Das Kind wardt todt von ihm geschnitten.»

Zuber (siehe Anm. 7), S.80. Nach einer Eintragung aus den Kirchengutsrechnungen von Sumiswald wurde dem «Möösli Schärer» eine neue Dublone für einen Kaiserschnitt bezahlt. Dem Preis nach war es eine erfolgreiche Operation, wenigstens für das Kind. (1 Dublone = ca. 384. Franken nach Umrechnungstabelle 1952/53 von Christian Lerch, Staatsarchiv Bern.)

18 Peter Schütz in Trachselwald «tractierte» im Winter 1680/81 einen jungen Uhrmacher fünf Monate und kurierte ihn von einer sonderbaren Krankheit. (Rubi, Christian, Bäder und Bader, «Bund», Nr. 199, 26. August 1978.)

1769 wurde dem Schärer Schütz in Grünen bei Sumiswald eine Patientin zur Kur ins Haus gegeben. Wenn ihm die Kur gelinge, so solle er 8 Kronen erhalten, wenn nicht, wöchentlich 15 Batzen, also entweder 400 Franken oder die Woche zu 30 Franken, nach Umrechnungstabelle 1952/53 (Zuber, Anm. 7, S.80).

Im Ratsmanual Burgdorf von 1787 lesen wir von einem Kind, das mit einer Krankheit behaftet war, die eine kräftige Kurart erfordere, und da sich der Chirurg Alexander Burger in Sumiswald zutraue, dieses Kind zu heilen, solle es ihm gebracht werden.

Michel Schüppach erreichte als Kurarzt einen ungewöhnlichen Ruf.

${ }^{19}$ Unterrichtsrodel Langnau 1630, Bd.3: Landsäckelmeisterrechnungen der Landschaft Emmental 1643 (Gemeindearchiv Langnau); Amtsrechnungen der Landvogtei Trachselwald 1645; Contraktenprotokolle Trachselwald (Grundbuchamt Trachselwald) 1771, Bd.52, S. 641; 1781, Bd. 70, S. 1153, enthalten in: Geschichte der Medizin im Emmental, S. 44, 45, 52, 53.

${ }^{20} \mathrm{Vgl}$. Anm. 19.

${ }^{21}$ Rennefahrt und Hintzsche (siehe Anm. 8), S. 97-100; 384f. 
22 Über Gemeindespitäler:

Anliker, Fritz, Von der Armenanstalt zum Pflegeheim, 75-Jahr-Jubiläum (Langnau) 1973; Gruner, Gottlieb Sigmund, Über Armenhäuser. In: Der Gemeinnützige Schweizer, Jg. 1, 1815;

Manuel, Carl, Jeremias Gotthelf, sein Leben und seine Schriften, Berlin 1858;

Vatter, Max, Das religiöse und kirchliche Leben, einschließlich des Fürsorgewesens. In: Heimatbuch des Amtes Burgdorf und der Kirchgemeinden Utzenstorf und Bätterkinden, 1938, S. 518-521;

Würgler, Hans, Heimatkunde von Rüegsau, Rüegsau 1965, S.33;

Zuber, Jakob, Die Armenanstalt der Einwohnergemeinde Sumiswald, 1812-1912, Sumiswald 1913.

${ }^{23}$ Dossier Notfallstuben, Sanitätswesen, Staatsarchiv Bern.

${ }^{24}$ Über das Bezirksspital in Burgdorf verwendete Unterlagen:

Kasser, Hermann, Das Bernbiet ehemals und heute, Bd.1, Bern 1905, S. 27.

Aus dem Spitalarchiv: Bericht der Delegiertenversammlung 1879; Jahresberichte von 1878 an.

25 Über das Bezirksspital in Sumiswald verwendete Unterlagen aus dem Spitalarchiv: Jahresberichte, Protokolle, Briefe, Rechnungen von 1880 an.

26 Über das Bezirksspital in Langnau verwendete Unterlagen aus dem Spitalarchiv: Rede zur Eröffnungsfeier eines neuen Gebäudes 1898, Bericht über die Krankenanstalt 1836-1907, Zeitungsberichte, Jahresberichte von 1898 an. (Die Jahresberichte enthalten bis 1940 reichere medizinische Aufschlüsse als seither.)

\section{Summary}

This paper deals with the medical provision for a country-people. It begins with the hospital of a religious order, with infirmaries and little monasteries in the Middle Ages. In the following centuries sick people - even the insanes - were lodged in families or private institutes of barber surgeons. The surgery reached good results in the 18th century. Stations for cases of necessity were the beginning of the later district hospitals. Some very good physicians worked there, e.g. Anton Fonio, who developed a method of blood conservation.

Marta Meyer-Salzmann

Ackersteinstraße 178

CH-8049 Zürich 